Itinéraires Itinéraires

Littérature, textes, cultures

\title{
Hugh Grady et Terence Hawkes (eds.), Presentist Shakespeares
}

Oxon, Routledge, coll. « Accents on Shakespeare », 2007.

\section{Anne-Valérie Dulac}

\section{(2) OpenEdition}

\section{Journals}

Édition électronique

URL : http://journals.openedition.org/itineraires/1766

DOI : 10.4000/itineraires. 1766

ISSN : 2427-920X

Éditeur

Pléiade

Édition imprimée

Date de publication : 1 décembre 2010

Pagination : 184-187

ISBN : 978-2-296-13183-5

ISSN : 2100-1340

Référence électronique

Anne-Valérie Dulac, « Hugh Grady et Terence Hawkes (eds.), Presentist Shakespeares », Itinéraires [En ligne], 2010-4 | 2010, mis en ligne le 01 décembre 2010, consulté le 22 septembre 2020. URL : http:// journals.openedition.org/itineraires/1766 ; DOI : https://doi.org/10.4000/itineraires.1766

Ce document a été généré automatiquement le 22 septembre 2020.

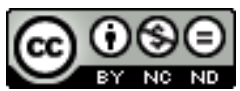

Itinéraires est mis à disposition selon les termes de la licence Creative Commons Attribution - Pas d'Utilisation Commerciale - Pas de Modification 4.0 International. 


\title{
Hugh Grady et Terence Hawkes (eds.), Presentist Shakespeares
}

Oxon, Routledge, coll. « Accents on Shakespeare », 2007.

\author{
Anne-Valérie Dulac
}

\section{RÉFÉRENCE}

Hugh Grady et Terence Hawkes (eds.), Presentist Shakespeares, Oxon, Routledge, coll.

« Accents on Shakespeare », 2007.

1 Les noms de Hugh Grady et Terence Hawkes, coéditeurs de Presentist Shakespeares ${ }^{1}$, sont très intimement associés au développement des études présentistes. Dès 1991, dans The Modernist Shakespeare ${ }^{2}$, Hugh Grady recommande à son lecteur de se saisir des «interconnections entre passé et présent $^{3}$ » au prix d'une prise de distance revendiquée avec le nouvel historicisme ${ }^{4}$ dont il tâche de démontrer les apories et les proximités paradoxales avec l'historicisme positiviste d'un E. Tillyard ${ }^{5}$. En 2000, Hawkes, en "président du présentisme ${ }^{6}$ ", établit une première synthèse de cette tendance dans Shakespeare in the Present ${ }^{7}$. Les articles rassemblés dans Presentist Shakespeares s'inscrivent dans la droite ligne de cette histoire, dont la voûte conceptuelle repose sur une clef centrale commune : le rejet du nouvel historicisme et de son pendant britannique, le matérialisme culturel.

2 Le présupposé initial du présentisme rejoint l'idée exprimée par Benedetto Croce que « $[\mathrm{t}]$ oute histoire digne de ce nom est histoire contemporaine ${ }^{8}$ ». Dans ce seul rappel gît, d'après nos auteurs, le grand écueil du nouvel historicisme. Dans le processus d'institutionnalisation qui lui a, d'après les auteurs, conféré une position quasi hégémonique, le nouvel historicisme aurait progressivement renoncé aux nuances du premier Greenblatt. L'ouverture de Renaissance Self-Fashioning formulait en effet un certain nombre de réserves relatives à la possibilité réelle d'engager un « dialogue avec les morts ${ }^{9} »$ et de se détacher tout à fait du présent de situation du critique ${ }^{10}$. 
Grady et Hawkes estiment que ces saines mises en garde liminaires de Greenblatt auraient été rapidement ensevelies sous la pratique néo-historiciste ultérieure qui n'aurait plus pour ambition que celle d'accumuler des faits, autant de "talismans ${ }^{11}$ " prélevés dans le champ de la culture matérielle et destinés à se prémunir, en la documentant, de la distance qui sépare le contemporain du early modern. À l'inverse, Grady et Hawkes réaffirment la précédence de la situation présente de réception du texte sur son historicité. Ce renversement informe leur choix de se saisir de l'adjectif "présentiste » réhabilité comme outil heuristique ${ }^{12}$. Mais ce terme, bien qu'il recouvre des aspirations communes à tous les contributeurs de Presentist Shakespeares, regroupe des approches très diverses. Au cours d'une table ronde virtuelle rassemblant, à l'instigation de Grady, bon nombre de contributeurs du volume quelques mois seulement après sa publication, Linda Charnes proposait d'envisager le présentisme comme "sensibilité » plutôt que comme "méthodologie ${ }^{13}$ ". Charnes estime que l'étiquette "présentiste» lui a d'ailleurs été attribuée sans qu'elle-même ne s'en revendique jamais. Michael Bristol donne dans le recueil un avis similaire. D'après lui, le concept de "présentisme ", dans la mesure où il est formé à partir du suffixe -isme qui le rapproche de théories dont Bristol tient à s'éloigner, ne convient que mal à ses propres intérêts. Les trois exemples convoqués afin d'illustrer la consubstantialité de l'«isme» et de trois dérives potentielles (l'hérésie, l'idéologie pernicieuse ou les errements intellectuels, illustrés respectivement par l'arminianisme, le nazisme, et l'historicisme), manifestent très symptomatiquement le rapport de certains présentistes au « démon de la théorie ${ }^{14}$ » dont le nouvel historicisme constitue à leurs yeux l'engeance récente la plus contestable.

4 Les deux premiers textes, signés de Terence Hawkes et de Catherine Belsey, offrent un diptyque introductif complémentaire sur la situation critique actuelle. Dans «Band of Brothers ", Hawkes revient sur l'origine relativement récente du "transatlantisme » shakespearien, issu du dépassement d'un vieux Kulturkampf de guerre froide dont Hawkes fait correspondre les moments et lieux de cristallisation à la lecture marxiste du Timon of Athens. Au contraire, la place occupée par le barde dans la diffusion actuelle du multiculturalisme témoigne d'après lui de l'émergence d'un nouveau Shakespeare "supra-politique ${ }^{15}$ ». Après Hawkes, Catherine Belsey fait porter sa réflexion sur la généalogie du nouvel historicisme et s'applique à en démontrer l'américanité propre, et, partant, son éloignement de la French Theory. Là où Hawkes engage à la fraternité transatlantique, Belsey ne manque pas de souligner l'impossible superposition des approches européennes et américaines, en s'appuyant sur les diverses résonances attachées, après 1945, au mot « résistance » de part et d'autre de l'Atlantique, ainsi que sur l'influence du fonctionnalisme américain de Parsons ou Fukuyama sur Greenblatt.

Une fois situé en regard du nouvel historicisme, et contre lui, le présentisme est alors décliné en sept articles portant chacun sur une pièce du corpus shakespearien : Michael Bristol, dans une démonstration qui n'est pas sans évoquer l'importance de la pédagogie et du travail en classe aux yeux des présentistes, propose de relire King Lear à l'aune de citations de travaux d'étudiants. Le nom de ces jeunes shakespeariens qui figurent à la fin de l'article en une colonne typographiquement évocatrice de la liste des personnages d'une pièce semble indiquer que King Lear se rejoue bel et bien dans chaque lecture, chaque année, dans chaque classe. Puis Linda Charnes se saisit d'Othello pour interroger les débats passés et actuels autour de la question du mariage interracial mais également les questions relatives à l'historicisme et au présentisme 
vues depuis la pièce et les images du « futur » qu'elle propose. John Drakakis, à l'étude du Merchant of Venice, envisage la portée centrale et toute problématique du travail d'édition, pour un présentiste attaché à reconnaître ses propres limites de reconstitution d'un texte original. Ewan Fernie s'intéresse à sa suite à l'écho actuel de la violence telle qu'elle est mise en scène dans l'action d'Henry $V$ et la grande place qu'elle ménage à la participation du spectateur. Dans l'article suivant, «Lavinia as "Blank Page" », Evelyn Gajowksi revient sur l'actualité du discours sur le viol porté par l'héroïne de Titus Andronicus qu'elle resitue dans la constitution historique du discours féministe critique.

6 Les deux derniers textes, enfin, à l'image des deux premiers, semblent dessiner une orientation critique commune plus nette, faisant ici office de conclusion. La contribution de Grady présente en effet une historiographie critique d'Hamlet tout entière dirigée vers l'exposition des limites de la lecture de la tragédie par Greenblatt réduite à une " œuvre de restauration culturelle incomplète ", simple " contribution à la culture du postmodernisme ${ }^{16} »$. Kiernan Ryan, dans le dernier article du volume, prolonge le plus fermement ce biais contre-historiciste. Troilus and Cressida offre d'après lui la preuve que ce n'est pas tant le présentisme que l'imagination shakespearienne même qui relèverait de l'anachronisme. En ce sens, les lectures féministes, marxistes ou déconstructionnistes seraient plus essentiellement historiques que tout ce que l'ancien comme le nouvel historicisme ont pu en dire. Presentist Shakespeares se clôt ainsi sur une reformulation explicite de l'esprit qui a présidé à sa publication: la volonté plus ou moins assumée des auteurs de rompre publiquement avec l'historicisme.

\section{NOTES}

1. En 2007, année de parution de Presentist Shakespeares, Hugh Grady engage d'ailleurs sur un site d'études shakespeariennes une discussion très fournie autour de la question du "présentisme ». Ces échanges sont consultables à l'adresse suivante: http://shaksper.net/archive/2007/248january/25262-shaksper-roundtable-presentism(consulté le 8 novembre 2010).

2. Hugh Grady, The Modernist Shakespeare: Critical Texts in the Material World, Oxford, Clarendon Press, 1991.

3. Ibid., p. 235.

4. Grady y formule une critique éloquente de Greenblatt: "[T]he totalizing tendencies of Greenblatt's theory and the doctrinaire residues of the Althusserian phase of British postAlthusserian Marxism suggest a potential for turning the new historicism into an ideological practice for a socially isolated and élitist professorate content to explain the powerlessness of everyone else as a bitter (non) acknowledgment of their/our own impotence » (ibid., p. 235).

5. Voir en particulier le chapitre "The New Historicism and Cultural Materialism » (ibid., p. 225-235).

6. L'expression est de John Drakakis, l'un des auteurs de Presentist Shakespeares, et apparaît dans la table ronde virtuelle consacrée au présentisme : http://shaksper.net/archive/2007/250-april/ 25468-shaksper-roundtable-presentism (consulté le 8 novembre 2010). 
7. Terence Hawkes, Shakespeare in the Present, Oxon, coll. « Accents on Shakespeare ", Routledge, 2002. Après avoir été General Editor de la collection « New Accents » chez Methuen, Hawkes a pris la direction de la collection "Accents on Shakespeare" pour Routledge créée en 1999. Cette collection lui permet, depuis près de dix ans, de faire connaître ses propres travaux ainsi que ceux d'autres représentants des études présentistes, dont le Presentist Shakespeares constitue le plus récent exemple. La plupart des contributeurs à cet ouvrage ont d'ailleurs déjà édité, dans cette collection, leurs propres textes (c'est le cas, notamment, de Ewan Fernie, Michael Bristol, ou Linda Charnes).

8. Benedetto Croce (1866-1952), Contributo alla critica di me stesso, Paris, Nagel, 1949, p. 110. Défenseur ardent de l'autonomie de l'art, Croce était également fin connaisseur de Shakespeare, ainsi qu'en témoigne son Arioste, Shakespeare et Corneille (1920). Ses positions, en termes d'histoire et d'analyses littéraires étaient fondées sur le refus du positivisme et l'impossibilité de bâtir une « histoire littéraire » reposant sur des prémisses historiographiques propres à d'autres champs historiques. Gramsci a d'ailleurs bâti en grande partie sa propre vision de l'historicisme en opposition à la pensée de Croce.

9. Cette expression est inspirée des premiers mots du Shakespearean Negotiations de Greenblatt: «I began with a desire to speak with the dead (Shakespearean Negotiations: The Circulation of Social Energy in Renaissance England, Berkeley, University of California Press, 1988).

10. "[I]f cultural poetics is conscious of its status as interpretation, this consciousness must extend to an acceptance of the impossibility of fully reconstructing and reentering the culture of the sixteenth century, of leaving behind one's own situation » (ibid., p. 5).

11. Hugh Grady et Terence Hawkes, Presentist Shakespeares, op. cit., p. 2.

12. Voir par exemple Grady : «Presentism has been up to now a pejorative term; it was coined to designate universalizing historical methodologies which denied historical difference and naively imposed their own concepts and rationality onto an understanding of the past. The term in that sense can still play a useful role as a pejorative, but in the present situation in the field, we need to re-define and transvalue it as a positive term, to designate methods which understand the limits of historicism, its inability to transcend our own situation, and the need to come to terms with the past from within our current, unique point in history » (http://shaksper.net/ archive/2007/248-january/25262-shaksper-roundtable-presentism), consulté le 8 novembre 2010.

13. « $[\mathrm{P}]$ resentism is not a methodology but rather a sensibility, one that always poses the following question: why should anyone care about this now? » (ibid.).

14. L'expression est inspirée du titre d'un ouvrage d'Antoine Compagnon (Le Démon de la théorie, Paris, Seuil, 2001) qui propose une lecture « française » très différente et bien moins catégorique que celle des présentistes des limites de la théorie en littérature.

15. L'expression est adaptée du titre de l'une des sous-sections de l'article, « above politics ».

16. Presentist Shakespeares, op. cit., p. 161.

\section{AUTEURS}

\section{ANNE-VALÉRIE DULAC}

Université Paris-Est Créteil Val de Marne 